\title{
Comparative efficacy of terbutaline sulphate delivered by Turbuhaler dry powder inhaler or pressurised metered dose inhaler with Nebuhaler spacer in children during an acute asthmatic episode
}

\author{
S Drblik, G Lapierre, R Thivierge, J Turgeon, P Gaudreault, B Cummins-McManus, \\ I Verdy, J Haddon, J Lee, S Spier
}

See end of article for authors' affiliations

Correspondence to Dr S Spier, Alberta Children's Hospital, 1820 Richmond Road, SW Calgary, Alberta, T2T 5C7

Canada;

sheldon.spier@

crha-health.ab.ca

Accepted

24 October 2002

\begin{abstract}
Aims: To compare the efficacy of terbutaline sulphate delivered via Turbuhaler with a pressurised metered dose inhaler (pMDI) connected to Nebuhaler spacer in a population of asthmatic children presenting to emergency departments because of an acute episode of asthma.

Methods: Randomised double blind, double dummy, parallel study of acute asthma in the emergency department. A total of 112 children (6-16 years), who had a diagnosis of asthma, a baseline FEV of $25-60 \%$ of predicted normal value (PNV), and the ability to perform spirometry were studied. Patients received two doses of $0.5 \mathrm{mg} / 10 \mathrm{~kg}$ (maximum $2.0 \mathrm{mg}$ ) of terbutaline sulphate at time 0 minutes and time 30 minutes. The two groups were also stratified into subgroups based on $\mathrm{FEV}_{1}: 25-45 \%$ and 45.1-60\% PNV. FEV, before treatment and at two 15-minute intervals after each treatment was the main outcome measure. PIF, PEF, heart rate, $\mathrm{SpO}_{2}$, and tremor were also measured at these times.

Results: Both the Turbuhaler and $\mathrm{pMDI}+$ Nebuhaler groups showed significant increases from baseline to final value in their $\mathrm{FEV}$, results, $49 \%$ and $50 \%$ change from baseline to $t=60 \mathrm{~min}$, respectively $(p<0.001)$ using last value carried forward. No significant difference was found between the two groups for these results. Subanalysis of the stratified groups revealed similar results. In addition, no significant difference was found in the group and subgroup comparisons for heart rate, $\mathrm{SpO}_{2}$, and tremor. Conclusion: Results show that Turbuhaler and $\mathrm{pMDI}+\mathrm{Nebuhaler}$ are similar in terms of benefit and side effects in the treatment of acute moderate to severe asthma attacks in this study population.
\end{abstract}

$\ldots \ldots \ldots \ldots \ldots \ldots$

(1)

$\mathrm{T}$ he conventional first line treatment of acute asthma for the paediatric population is a $\beta_{2}$ agonist administered in an aqueous solution by nebulisation. In recent years, $\beta_{2}$ agonists given by pressurised metered dose inhalers plus a spacer have been shown to be as efficacious as when given via a nebuliser to children with acute asthma. ${ }^{1-5}$ These findings suggested that other treatment modalities could offer the same advantages and be used as alternatives in the emergency setting in treating patients with acute asthma.

Dry powder formulations of $\beta_{2}$ agonists are a well established treatment of children with clinically stable asthma and have been shown to be as efficacious as the pressurised metered dose inhaler (pMDI) with a spacer. ${ }^{6-9}$ Only recently, the use of dry powder inhalers has been investigated as a possible alternative for acute asthma; however, these studies generally have been in adults. ${ }^{11}$ To our knowledge, only two investigations to date have attempted to address this question in the paediatric population using a limited number of subjects. ${ }^{12}{ }^{13}$ The reluctance to use an effort dependent breath actuated device in an acute setting may be due, in part, to the belief that either inspiratory flow is sufficiently compromised during an acute exacerbation or children may not be able to perform the inspiratory manoeuvre correctly, thereby affecting the amount of medication being effectively delivered.

We designed this study, therefore, to compare one such dry powder inhaler (DPI), Turbuhaler (AstraZeneca), with a pMDI and a spacer, Nebuhaler (AstraZeneca), in their efficacy of delivering equally effective doses of terbutaline sulphate to children presenting to the emergency department for first line treatment of an acute asthmatic episode.

\section{METHODOLOGY \\ Patients}

Patients aged 6-16 years inclusive, with acute asthma, who presented to one of three emergency units in Canada, were recruited to the study: Hôpital Ste-Justine, Montréal, Québec $(\mathrm{n}=102)$; Alberta Children's Hospital, Calgary, Alberta $(\mathrm{n}=7)$; and Misericordia Community Hospital, Edmonton, Alberta $(n=3)$. Inclusion criteria were a baseline forced expiratory volume in one second $\left(\mathrm{FEV}_{1}\right)$ of $25-60 \%$ of predicted normal value (PNV) ${ }^{14}$ baseline peak inspiratory flow (PIF) of at least $30 \mathrm{l} / \mathrm{min}$ when measured through Turbuhaler, and the ability to perform correctly both spirometry and the inhalational techniques required of Turbuhaler and pMDI+Nebuhaler. In addition, baseline resting heart rate could not exceed 160 beats/minute.

Patients were ineligible if they had used inhaled $\beta_{2}$ agonists within three hours prior to enrolment, or theophylline, ipratropium bromide, or oral $\beta_{2}$ agonists within 12 hours. Potential patients were also excluded if they had any known contraindication to sympathomimetic amines, lactose (non-drug component of the placebo), or pMDI additives (Freon 11, 12, 114, and sorbitan trioleate).

The respective ethics committee of each participating hospital approved the study, and informed written consent was obtained from the parents. The study was performed in accordance with

Abbreviations: DPI, dry powder inhaler; $\mathrm{FEV}_{1}$, forced expiratory volume in one second; ITT, intention to treat; PEF, peak expiratory flow; PIF, peak inspiratory flow; $\mathrm{pMDI}$, pressurised metered dose inhaler; PNV, predicted normal value; $\mathrm{SpO}_{2}$, transcutaneous oxygen saturation; t, time 
the principles stated in the Guidelines on Research Involving Human Patients issued by the Medical Research Council of Canada, which encompass the Declaration of Helsinki of 1975 (revised 1983), and with Good Clinical Practice.

\section{Study design}

A double blind, double dummy, randomised, parallel design was used. Patients were first stratified into two groups based on their initial $\mathrm{FEV}_{1}$ expressed as the percent PNV: 25-45\% and $45.1-60 \%$. In addition, within each stratified group the treatment and delivery system were randomised and were alternated to avoid a potential bias based on the order of treatment and device.

Patients were randomised into two groups to receive either $0.5 \mathrm{mg} / 10 \mathrm{~kg}$ (maximum $2.0 \mathrm{mg}$ ) terbutaline sulphate via Turbuhaler and placebo pMDI+Nebuhaler spacer (or the reverse order) or $0.5 \mathrm{mg} / 10 \mathrm{~kg}$ (maximum $2.0 \mathrm{mg}$ ) terbutaline sulphate via the pMDI+Nebuhaler spacer and placebo Turbuhaler (or the reverse order). The treatment was given twice within the 60 minute treatment period, once at the beginning of the study, $\mathrm{t}=0$, and once again after 30 minutes, $\mathrm{t}=30$. Approximately 10 minutes prior to commencing the study, each patient received a dose of oral prednisone. Patients weighing $<25.0 \mathrm{~kg}$ received $25 \mathrm{mg}$ prednisone, while patients weighing $\geqslant 25.0 \mathrm{~kg}$ received $50 \mathrm{mg}$.

All patients were shown the required inhalation techniques for each device and were required to show the techniques prior to commencing the study. For Turbuhaler, patients were instructed to inhale as fast and as deeply as possible from functional residual capacity followed by a natural breath hold lasting approximately three seconds. ${ }^{15}$ For the pMDI+Nebuhaler spacer, five breaths of sufficient force to move the valve in Nebuhaler were required for each puff from the pMDI. ${ }^{16}$ All treatments were given with the patients in the sitting position.

\section{Measurements}

Spirometry was performed on a Vitalograph Compact II to measure the variables peak inspiratory flow (PIF) and FEV . Three technically correct forced expiratory manoeuvres were executed from which the best result was chosen, in accordance with all but one of the American Thoracic Society guidelines. ${ }^{17} \mathrm{~A}$ difference of $8 \%$ instead of $5 \%$ in $\mathrm{FEV}_{1}$ among the three test results was accepted since the acute state of the patient would not permit excessive manoeuvres for fear of risking bronchospasm or attrition.

Measurements of PIF, both with and without Turbuhaler, were also made in triplicate using the Micro Plus Spirometer modified for inspiratory flow. Peak inspiratory flow through Turbuhaler was obtained by attaching Turbuhaler in series to the spirometer by a specially designed adapter. Recordings of PIF through Turbuhaler were obtained during treatment delivery at $\mathrm{t}=0$ minutes and $\mathrm{t}=30$ minutes. All other spirometry assessments were completed at baseline and following both treatments at 15 minute intervals, which resulted in recordings at time $0,15,30,45$, and 60 minutes.

Heart rate and $\mathrm{SpO}_{2}$ were measured using a pulse oximeter. Hand tremor was assessed using predefined criteria and recorded as absent, mild, moderate, or severe. The data were collected from each patient prior to spirometry, with the patient having assumed a semirecumbent position for at least five minutes.

The right to terminate the study before completion was an option that the patient, parent, or physician could exercise at any time. In addition, patients were withdrawn from the study if (1) their baseline $\mathrm{FEV}_{1}$ did not increase by more than $10 \% 30$ minutes after the first treatment; (2) their baseline heart rate increased by more than $40 \%$; or (3) their tremor was assessed as severe.

\section{Statistical analysis}

The data were analysed with SAS V6.12/Windows NT. The change from baseline to the last $\mathrm{FEV}_{1}$, PIF with and without
Turbuhaler, PEF, and safety variables (heart rate, tremor, and $\mathrm{SpO}_{2}$ ) were compared between the treatment groups by an analysis of covariance (ANCOVA) with factors for treatment, stratification, and interaction effects between treatment and stratification in the preliminary model. The corresponding baseline value was used as the covariant. Where spirometry data were missing, the principle of last value carried forward was used. The interaction effect was removed from the final model because of statistical insignificance. The 95\% confidence intervals for treatment differences were calculated. No analysis for centre effect was undertaken because of the small sample sizes at two of the three centres.

The sample size calculation was based on comparison of the mean change in FEV \%PNV from baseline between treatments. With a two sided test at $\alpha=0.05$ and a power of $80 \%$, 64 patients were required in each group in order to detect a clinically relevant difference of $5 \%$ in the $\mathrm{FEV}_{1}$ with a standard deviation (SD) of $10 \%$. Since the study was terminated prematurely because of a slow recruitment rate, a retrospective sample size calculation was performed. The actual power of the study was $75 \%$ given a total of 112 patients and SD of $10 \%$. All randomised patients were included in the intention to treat analysis (ITT). All statistical tests were two sided and $p$ values of $<0.05$ were considered statistically significant.

\section{RESULTS}

Of the 112 patients that were enrolled into the study, nine children did not complete the investigation. Six patients were from the Turbuhaler group, of which three were unwilling to continue the study and three were removed after 30 minutes when their baseline FEV 1 failed to increase by more than $10 \%$. Their ages ranged from 6 to 10 years, and their baseline FEV values were $36-60 \%$ PNV. The reasons for early withdrawal for the three patients in the pMDI+Nebuhaler group were unwillingness to continue in two patients and a $<10 \%$ increase in $\mathrm{FEV}_{1}$ after 30 minutes following the first treatment in one patient. These three patients were between 10 and 13 years of age with baseline FEV values $28-53 \%$ PNV. Since the ITT approach was selected for analysing the data, all data were included in the analysis.

Table 1 shows the demographic and baseline spirometric data of the 112 patients who were enrolled in the study. No significant difference was found between the two groups for any baseline variable, indicating that both groups were well matched for sex, age, height, and $\mathrm{FEV}_{1}$. The mean baseline FEV (SD) value for the Turbuhaler group was $44.8 \%$ (9.0\%) PNV, and the mean baseline $\mathrm{FEV}_{1}$ (SD) value for the pMDI+Nebuhaler group was $43.4 \%(10.1 \%)$ PNV. Stratification of the two treatment arms into the two subgroups $\left(\mathrm{FEV}_{1} 25-45 \%\right.$ and $45.1-60 \%$ ) resulted in comparable findings. In the FEV $25-45 \%$ stratum, there were 26 patients in the Turbuhaler group $\left(\mathrm{FEV}_{1}\right.$ \%PNV: 36.6 (4.9); range 26-44) and 26 patients in the pMDI+Nebuhaler group (FEV ${ }_{1}$ PNV: 34.1 (5.1); range 26-45). In the $\mathrm{FEV}_{1} 45.1-60 \%$ stratum, there were 31 patients in the Turbuhaler group (FEV, \%PNV: 51.6 (5.0); range 43-60) and 29 in the pMDI+Nebuhaler group (FEV ${ }_{1} \% \mathrm{PNV}$ : 51.8 (4.4); range 46-60). Neither these differences nor any other baseline measurements resulting from the stratification proved to be significantly different between the two groups.

Similar doses of terbutaline sulphate were given to both groups at the two dosing times. At the initial treatment time $(t=0)$, the Turbuhaler group received a mean value of 1.75 (0.33) $\mathrm{mg}$ (range 1.0-2.0 mg), while the pMDI+Nebuhaler group received a mean value of $1.71(0.3) \mathrm{mg}$ (range 1.0-2.0 $\mathrm{mg}$ ) of terbutaline sulphate. At the second treatment time $(t=30)$, the Turbuhaler group received an additional mean amount of $1.78(0.32) \mathrm{mg}$ (range $1.0-2.0 \mathrm{mg}$ ), and the pMDI+Nebuhaler group received a mean amount of 1.69 (0.33) $\mathrm{mg}$ (range 1.0-2.0 mg). None of these differences were significantly different between the two groups. 


\begin{tabular}{lll} 
Table 1 & Demography of patient population \\
\hline & Turbuhaler & $\begin{array}{l}\text { pMDI } \\
\text { +Nebuhaler }\end{array}$ \\
\hline $\mathrm{n}$ & 57 & 55 \\
Male/female & $35 / 22$ & $39 / 16$ \\
Age (y) & $10.7(2.9)[6-16]$ & $10.3(2.8)[6-16]$ \\
Height (cm) & $145.6(15.2)$ & $143.0(14.6)$ \\
Weight (kg) & {$[114-183]$} & {$[121-175]$} \\
FEV $_{1}(\mathrm{ll})$ & $42.0(16.7)$ & $40.0(14.2)$ \\
FEV $_{1}$ (\%PNV) & {$[20-120]$} & {$[21-76]$} \\
Receiving IGCS & $1.08(0.36)$ & $0.98(0.34)$ \\
Receiving short acting $\beta_{2}$ & {$[0.43-1.90]$} & {$[0.50-1.81]$} \\
agonists & {$[25.8(9.0)$} & $43.4(10.1)$ \\
Withdrawn & $49.60 .4]$ & {$[25.7-59.8]$} \\
\hline
\end{tabular}

Results expressed as mean (SD) [range] where appropriate. IGCS, inhaled glucocorticosteroids; pMDI, pressurised metered dose inhaler.

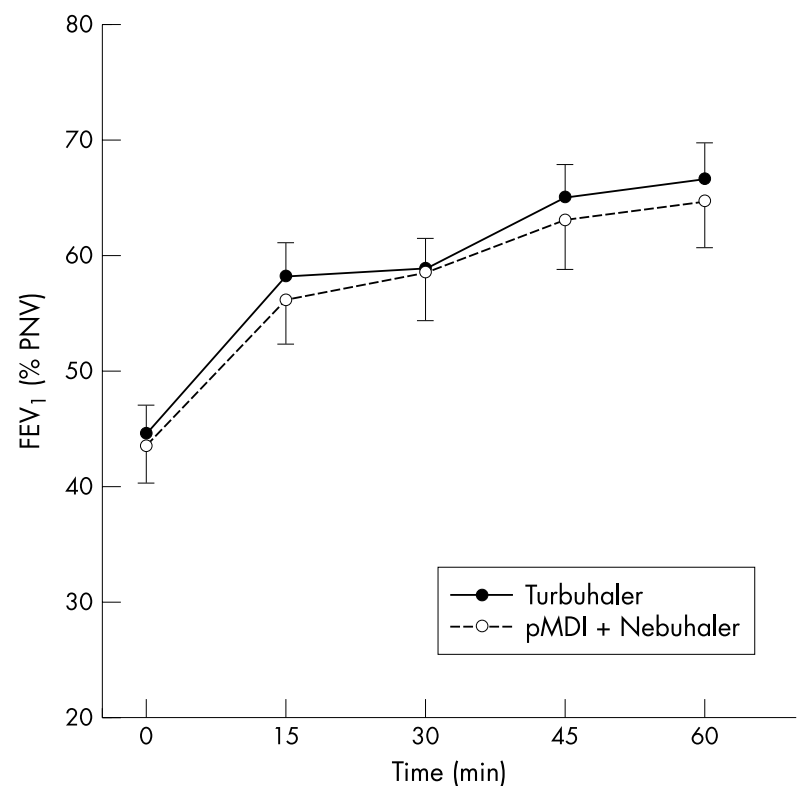

Figure $1 \mathrm{FEV}, \%$ PNV over time (mean $\pm 2 \mathrm{SEM}$ ); $p<0.05$ change from baseline to last value within each group. The differences between groups were not significant.

Comparison between the two groups showed no significant difference for the changes in $\mathrm{FEV}_{1}$, the primary variable, from baseline to final value ( $\mathrm{p}>0.05 ; 95 \% \mathrm{CI}-4.4 \%$ to $3.5 \% \mathrm{PNV}$ ). Each group did show, however, a significant improvement after each treatment when compared with baseline values $(p<0.001)$. As fig 1 shows, 30 minutes following the first treatment dose at $\mathrm{t}=0$, there was a $33 \%$ increase in the $\mathrm{FEV}_{1}$ \%PNV in the Turbuhaler group and a 35\% increase in the pMDI+Nebuhaler group. After the second and final treatment dose given at $\mathrm{t}=30$, additional increases of $21 \%$ and $16 \%$ were found in the Turbuhaler and Nebuhaler groups respectively, resulting in an overall change from baseline to final value of approximately $49 \%$ for the Turbuhaler group and $50 \%$ for the Nebuhaler group.

Figure 2 represents the mean $\mathrm{FEV}_{1} \%$ PNV for the two groups stratified into their two subgroups according to baseline FEV $_{1} \%$ PNV: $25-45 \%$ and $45.1-60 \%$. There was no significant difference in baseline values between the two groups within the $25-45 \%$ stratum, and the final mean value after the two treatment doses at $\mathrm{t}=60$ was $62.1 \%$ (12.3\%) PNV for the Turbuhaler group and $55.7 \% \quad(13.0 \%)$ PNV for the pMDI+Nebuhaler group. This results in a mean absolute

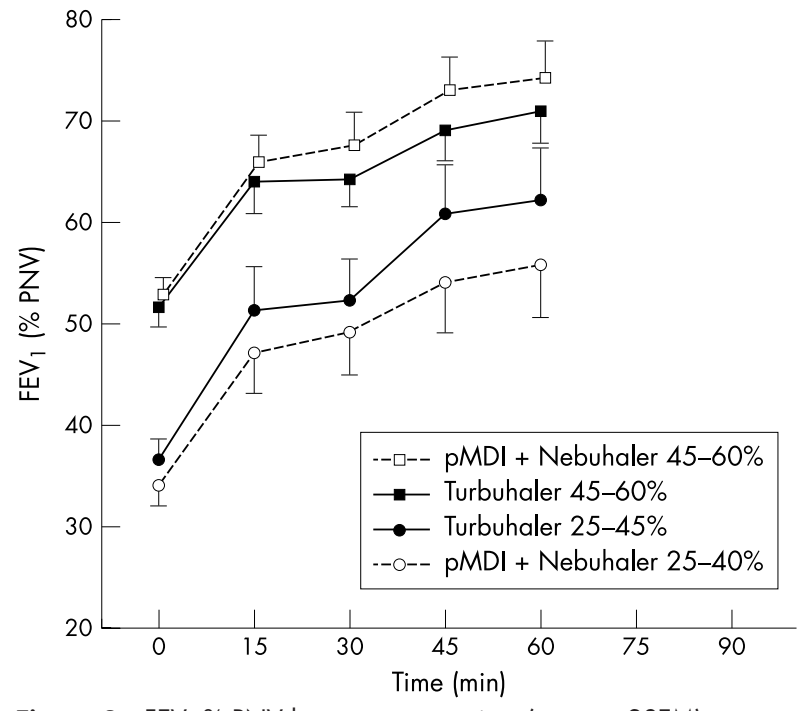

Figure $2 \mathrm{FEV}, \% \mathrm{PNV}$ by stratum over time (mean $\pm 2 \mathrm{SEM}$ ).

change of $26 \%$ and $22 \%$, respectively. While these increases were statistically significant within each treatment stratum, no difference was observed between the Turbuhaler and the pMDI+ Nebuhaler within the $25-45 \%$ stratum. Similar statistical results were found for the $45.1-60 \%$ stratum. The final mean value at $\mathrm{t}=60$ minutes was $70.8 \%(8.1 \%)$ PNV for the Turbuhaler group and $73.1 \% \quad(8.8 \%)$ PNV for the pMDI+Nebuhaler group, which yielded a mean absolute change of $19 \%$ and $21 \%$, respectively. Once again, although these increases proved to be statistically significant within each treatment subgroup, no difference was found between the Turbuhaler and pMDI+Nebuhaler $45.1-60 \%$ stratum. Similar statistical results were found for PIF and PEF.

Figure 3 shows the relation between the bronchodilatory effects of the terbutaline sulphate delivered in dry powder and the inspiratory flow through Turbuhaler. Only the data from the Turbuhaler group are shown. No significant correlation could be found between the percent change from baseline to the final $\mathrm{FEV}_{1}$ value and the PIF measured through Turbuhaler during the first treatment dose at $\mathrm{t}=0(\mathrm{p}=0.10 ; r=0.31)$. Similar results were found when this relation was examined using the PIF measured through Turbuhaler during the second treatment dose at $t=30$. The six data points below the $10 \%$ mark on the $y$ axis are the results of the six patients who were enrolled and randomised into the Turbuhaler group but did not complete the protocol.

The changes for heart rate from baseline to final value were statistically significant $(\mathrm{p}<0.001)$ within each group, but no significant difference was found when between group comparisons were made (table 2). The greatest increase in heart rate for both groups occurred 30 minutes after the first treatment (Turbuhaler: 12\% change; pMDI+Nebuhaler: 10\% change) which was followed by another increase, albeit smaller, 30 minutes after the second treatment (Turbuhaler and pMDI+Nebuhaler: $7 \%$ change). No patient in either group had an increase in heart rate of more than $40 \%$ above their baseline value. The changes in $\mathrm{SpO}_{2}$ from baseline to final value were not significantly different between the two groups, and although within group comparisons were statistically significant, there was no clinical relevance to these changes.

On entry into the study, $88 \%$ of the Turbuhaler patients and $91 \%$ of the pMDI + Nebuhaler patients did not exhibit any hand tremor. At the final measurement interval of the study, after two treatment doses had been given, mild hand tremor was observed in $26 \%$ and $56 \%$ of the Turbuhaler and pMDI+Nebuhaler patients, respectively, while $61 \%$ of the Turbuhaler and $40 \%$ of the pMDI+Nebuhaler patients were 


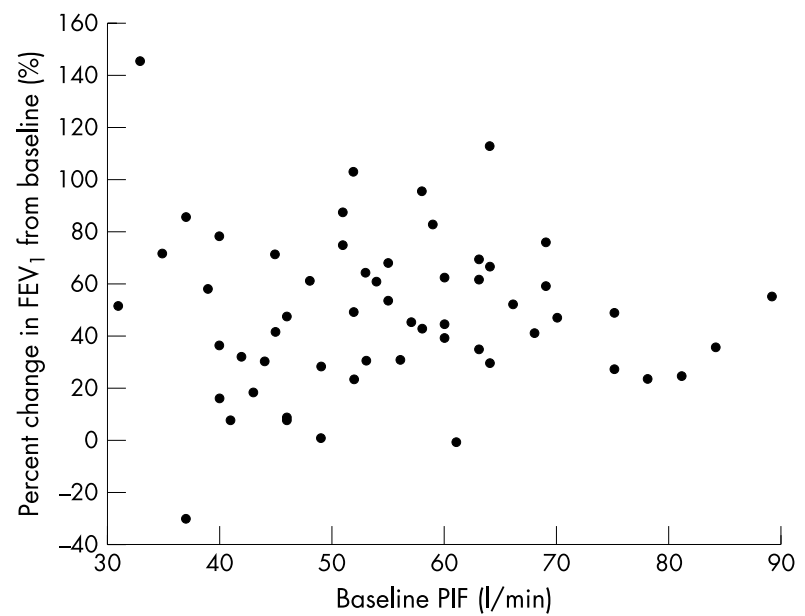

Figure 3 Percent change in FEV, versus baseline PIF via Turbuhaler device for the Turbohaler group.

exhibiting moderate hand tremor. Statistical analysis of these differences did not show any significance for the between group comparisons, while within group comparisons did reach significance $(\mathrm{p}<0.001)$. At no time during the study did any patient exhibit a severe hand tremor.

\section{DISCUSSION}

The results from this study have shown that in our sample population terbutaline sulphate delivered in dry powder by Turbuhaler was as efficacious as the same nominal dose of terbutaline sulphate delivered by the pMDI+Nebuhaler spacer as a first line treatment of moderate and severe acute asthma attacks in children. These findings are in agreement with previous reports that suggested that Turbuhaler was an effective method of treating children during an acute asthma exacerbation. ${ }^{12}{ }^{13}$ Springer and colleagues ${ }^{13}$ compared $2.5 \mathrm{mg}$ terbutaline sulphate delivered via Turbuhaler with $5.0 \mathrm{mg}$ terbutaline sulphate given by nebulisation and found significant and comparable improvements in PEF in a sample of 12 children (aged 7-12 years) attending an emergency department for treatment of an acute asthma exacerbation. In the other paediatric study, Rufin and colleagues $^{12}$ recruited 30 children (aged 6-14.5 years) with moderate acute asthma from an outpatient clinic. Using an open design, two groups of 15 patients received a single dose of $0.5 \mathrm{mg}$ terbutaline sulphate either via Turbuhaler or by a pMDI+Nebuhaler. This study also found significant and comparable improvements in $\mathrm{FEV}_{1}$ between the two groups up to 30 minutes following the treatment.

While the conclusions of these studies are in agreement with our study, neither study reported their statistical power. Therefore, the possibility exists that their sample sizes were too small to detect a significant treatment difference. Although the actual power of the current study was calculated to be $75 \%$ instead of the initially planned $80 \%$, this was not a concern since the confidence interval of the treatment difference had a small range (that is, both the lower and upper limits were less than $5 \%$ ). If we had chosen a value of $7 \%$ instead of $5 \%$ for the clinically relevant difference in $F E V_{1}$ as significant, the power of our study would increase to $96 \%$. In fact, a clinically relevant difference as high as $12 \%$ in the FEV PNV has been used previously in a comparably designed emergency department study which examined the difference between $\beta_{2}$ agonists delivered by nebuliser and pMDI with a spacer in children with mild to severe acute asthma. ${ }^{5}$ In addition, we chose to compare the Turbuhaler with a metered dose inhaler and spacer because of the simplicity and ease of use of the spacer, as well as results of numerous studies, including that of Kerem and colleagues, ${ }^{5}$ showing the equivalency of the spacer to the wet nebuliser.

The lack of correlation between the percent increase in $\mathrm{FEV}_{1}$ from baseline to final value and the measured inspiratory flow through Turbuhaler (fig 3) suggests that the bronchodilatory effects achieved with the DPI were independent of inspiratory flow above our minimum requirement of $30 \mathrm{l} / \mathrm{min}$. Similar observations were reported by Pedersen and colleagues. ${ }^{18}$ Their investigation examined the bronchodilatory effects of a submaximal dose of terbutaline sulphate delivered via Turbuhaler while varying inspiratory flows between 13 and $60 \mathrm{l} / \mathrm{min}$ in a sample of 14 children (aged 7-15 years) with stable chronic asthma. They found the increase in FEV 1 after treatment was comparable for patients with mean inspiratory flows of 31-60 $\mathrm{l} / \mathrm{min}$. However, when the inspiratory flow was decreased to 22 $\mathrm{l} / \mathrm{min}$., the treatment effect was reduced by approximately $67 \%$. In addition, a further decrease in inspiratory flow to $13 \mathrm{l} / \mathrm{min}$ resulted in a $33 \%$ treatment effect. Based on their findings, we chose a minimum inspiratory flow through Turbuhaler of 30 $\mathrm{l} / \mathrm{min}$ as an inclusion criterion, and it would appear that our results in an unstable acute asthmatic state are in agreement with their results in a stable chronic asthmatic state.

In our study, we did not find inspiratory flow to be a limiting factor in either patient attrition or recruitment. Once enrolled, no patient was withdrawn from the study because of their inability to continue to generate throughout the course of the protocol the minimally required inspiratory flow through Turbuhaler. This was observed in children as young as 6 years and in children with a baseline $\mathrm{FEV}_{1}$ as low as $25 \%$. In addition, apart from the 112 patients enrolled, only five patients (4\%), aged 6-13 years, whose baseline $\mathrm{FEV}_{1} \%$ PNV was $25-60 \%$, were not enrolled when they failed to achieve a Turbuhaler PIF of at least $30 \mathrm{l} / \mathrm{min}$; values were $24-28 \mathrm{l} / \mathrm{min}$. In this very small group, this was a result of either limited ability to perform the technique or actual flow limitation, and does not necessarily mean they would not have improved using this technique. This small number of subjects not studied should not detract from the major findings of this study. Of interest, studies investigating Turbuhaler in adults also found that only $2-4 \%$ of their sample were unable to generate $30 \mathrm{l} / \mathrm{min}$, and none of them were lower than $25 \mathrm{l} / \mathrm{min} .{ }^{11}{ }^{19}$ Although these patients were not

Table 2 Oxygen saturation and heart rate data shown by group and time post-treatment

\begin{tabular}{llllll}
\hline & \multicolumn{2}{l}{$\mathrm{SpO}_{2}(\%)$} & & \multicolumn{2}{l}{ Heart rate $($ beats $/$ min) } \\
\cline { 2 - 3 } \cline { 5 - 6 } Time $(\mathrm{min})$ & Turbohaler & $\mathrm{pMDI}+$ Nebuhaler & & Turbohaler & $\mathrm{pMDI}+$ Nebuhaler \\
\hline 0 & $95.5(0.5)$ & $95.2(0.5)$ & $103.1(4.1)$ & $102.8(3.6)$ \\
15 & $95.8(0.5)$ & $95.6(0.5)$ & & $113.1(5.3)$ & $112.5(4.4)$ \\
30 & $95.8(0.6)$ & $95.6(0.6)$ & $114.6(4.9)$ & $113.3(4.4)$ \\
45 & $96.5(0.6)$ & $95.8(0.6)$ & & $123.8(4.9)$ & $120.0(4.7)$ \\
60 & $96.5(0.5)$ & $96.0(0.6)$ & & $123.2(4.8)$ & $120.9(4.5)$ \\
\hline
\end{tabular}

The change from baseline to last value within each group was $p<0.05$. The differences between groups were not significant.

$\mathrm{SpO}_{2}$ and heart rate results are expressed as mean (2 SEM). 
enrolled in either their studies or in our study, it is quite probable that a beneficial response from the treatment would have been observed based on the findings from Pedersen and colleagues ${ }^{18}$ which showed that a significant bronchodilatation could still be achieved with Turbuhaler inspiratory flows of 22 $\mathrm{l} / \mathrm{min}$.

The similar bronchodilatory improvements accounted for by the changes in $\mathrm{FEV}_{1}$ not only were observed between the two groups, but also were comparable within the strata subgroups. The results of stratification of the sample into severe $(25-45 \%$ $\mathrm{FEV}_{1} \mathrm{PNV}$ ) and moderate (45.1-60\% $\mathrm{FEV}_{1} \mathrm{PNV}$ ) indicated that the bronchodilatory benefits were not related to degree of severity. Indeed, the mean absolute changes within each stratum were similar. However, when calculated on a relative basis, improvements in the severe stratum were greater than those changes calculated for the moderate stratum. One possible explanation for this observation could be that the severe patients had a greater potential to improve based on their lower initial value. The profile of our sample population could also account for the variation in bronchodilatory response. The duration of the asthmatic episode previously prescribed, type and dose of medication, and effective treatment delivery modalities were uncontrolled factors, which could have accounted for the degree of response to our treatment. Our patients were selected essentially on degree of severity, capabilities, and previous medication restrictions.

The calculated dose of terbutaline sulphate used for each patient was the same for the two delivery methods, and based on the FEV 1 results, provided similar bronchodilatory effects. On this topic the literature is equivocal. Previous cumulative dose response adult ${ }^{20}$ and children ${ }^{6} 9$ studies, as well as investigations comparing predetermined equivalent doses at various time intervals ${ }^{7821}$ have found no significant difference in efficacy between Turbuhaler and pMDI. On the other hand, other well designed studies have shown a higher dose-potency relation for Turbuhaler. ${ }^{1022-24}$ Moreover, Nana et al found that in their adult emergency unit study there was equal efficacy between the Turbuhaler and pMDI with Volumatic spacer, even though the pMDI contained twice the dose of salbutamol as did the Turbuhaler. ${ }^{24}$ The lack of agreement among the studies may be explained by their methodological differences. In our study, it was not our intent to evaluate a dose response to terbutaline sulphate but to initiate an effective treatment for an acute asthmatic episode based on our emergency department procedure which is to give a high dose of a $\beta_{2}$ agonist. The possibility exists therefore that our initial dose brought our patients nearer the flatter portion of the dose-response curve where differences between methods would not be detected.

Finally, our findings showed that the treatment benefits to side effects ratio were comparable between the two groups. The equal bronchodilatation, as indicated by the $\mathrm{FEV}_{1}$ results, achieved with both devices was accompanied by similar increases in heart rate and tremor within both groups. In addition to the finding that no patient showed either a $40 \%$ increase in baseline heart rate or a severe tremor during the treatment protocol, no patient requested to be withdrawn from the study because of an intolerance of these effects.

\section{Conclusion}

Terbutaline sulphate delivered in dry powder formulation via Turbuhaler was as efficacious as the pMDI+Nebuhaler spacer in the treatment of moderate to severe acute asthma attacks in our sample of children presenting to the emergency department. This observation was seen in children as young as 6 years of age, as well as in patients with an $\mathrm{FEV}_{1}$ percent PNV as low as $25 \%$, both factors not affecting their ability to perform correctly the inspiratory manoeuvre required of Turbuhaler. Our findings suggest that the Turbuhaler (DPI) is an effective alternative modality for the first line treatment of an acute asthmatic episode in children.

\section{ACKNOWLEDGEMENTS}

We wish to express our gratitude to Drs Benoit Bailey, Sylvie Bergeron, Perrette Payée, David Schragge, and Cheri Nijssen-Jordan for their assistance in the recruitment phase of the study. We would also like to acknowledge the support of AstraZeneca Canada, Inc.; Mr Michael Bukoski for help in preparing the protocol; and Dr Robert Jenkins for assistance in reviewing and preparing the manuscript.

\section{Authors' affiliations}

S Drblik, G Lapierre, Service of Pulmonology, Hôpital Ste-Justine, Université de Montréal, Québec, Canada

R Thivierge, J Turgeon, P Gaudreault, B Cummins-McManus,

I Verdy, Department of Pediatrics, Hôpital Ste-Justine

J Haddon, J Lee, AstraZeneca Canada, Inc.

S Spier, Respiratory Division, Alberta Children's Hospital, University of Calgary, Calgary, Alberta, Canada.

Investigator initiated trial supported by AstraZeneca Canada Inc. and La Société de pneumologie pédiatrique

\section{REFERENCES}

1 Freelander $\mathbf{M}$, van Asperen PP. Nebuhaler versus nebulizer in children with acute asthma. BM 1984;288:1873-4.

2 Fuglsang G, Pedersen S. Comparison of Nebuhaler ${ }^{\circledR}$ and nebulizer treatment of acute severe asthma in children. Eur J Respir Dis 1986;69:109-13.

3 Pendergast J, Hopkins J, Timms B, et al. Comparative efficacy of terbutaline administered by Nebuhaler ${ }^{\circledast}$ and by nebulizer in young children with acute asthma. Med J Aust 1989;151:406-8.

4 Idris $\mathbf{A H}$, McDermott MF, Raucci JC, et al. Emergency department treatment of severe asthma: metered-dose inhaler plus holding chamber is equivalent in effectiveness to nebulizer. Chest 1993:103:665-72.

5 Kerem E, Levison H, Schuh S, et al. Efficacy of albuterol administered by nebulizer versus spacer device in children with acute asthma. J Pediatr 1993; 123:313-17.

6 Fuglsang G, Pedersen S. Comparison of a new multidose powder inhaler with a pressurized aerosol in children with asthma. Pediatr Pulmonol 1989;7:1 12-15

7 Hultquist C, Ahlström H, Kjellman NI, et al. A double-blind comparison between a new multi-dose powder inhaler (Turbuhaler ${ }^{(B)}$ ) and metered dose inhaler in children with asthma. Allergy 1989;44:467-70.

8 Ahlström H, Svenonius E, Svensson M. Treatment of asthma in pre-school children with inhalation of terbutaline in Turbuhaler compared with Nebuhaler. Allergy 1989;44:515-18.

9 Laberge S, Spier S, Drblik SP, et al. Comparison of inhaled terbutaline administered by either the Turbuhaler dry powder inhaler or a metered-dose inhaler with spacer in preschool children with asthma. J Pediatr 1994;124:815-17.

10 Tönnesen F, Laursen LC, Evald T, et al. Bronchodilating effect of terbutaline powder in acute severe bronchial obstruction. Chest 1994: 105:697-700.

11 Engel T, Heinig JH, Madsen F, et al. Peak inspiratory flow and inspiratory vital capacity of patients with asthma measured with and without a new dry-powder inhaler device (Turbuhaler ${ }^{\circledR}$ ). Eur Respir J 1990;3:1037-41

12 Rufin P, Benoist MR, Panqueva $\mathrm{P}$, et al. Efficacité d'un inhalateur de poudre (Turbuhaler $\left.{ }^{\circledR}\right)$ dans la crise d'asthme modérée de l'enfant. Rev Mal Resp 1993;10:545-50

13 Springer C, Ståhl E, Schachter J, et al. Terbutaline given via Turbuhaler and in nebulised form in children with acute asthma. Eur J Clin Res 1996:8:163-9.

14 Polgar G, Promadhat V. Pulmonary function testing in children: techniques and standards. Philadelphia: WB Saunders Co., 1971

15 Hansen OR, Pedersen S. Optimal inhalation technique with terbutaline Turbuhaler. Eur Respir J 1989;2:637-9.

16 Gleeson JGA, Price JF. Nebuhaler technique. Br J Dis Chest 1988;82:172-4.

17 American Thoracic Society. Standardization of spirometry: 1994 update. Am J Respir Crit Care Med 1995;152:1 107-36.

18 Pedersen S, Hansen OR, Fuglsang G. Influence of inspiratory flow rate upon the effect of a Turbuhaler. Arch Dis Child 1990;65:308-10.

19 Brown PH, Ning ACWS, Greening AP, et al. Peak inspiratory flow through Turbuhaler ${ }^{\circledR}$ in acute asthma. Eur Respir J 1995;8:1940-1.

20 Persson G, Gruvstad E, Ståhl E. A new multiple dose powder inhaler $\left(\right.$ Turbuhaler $\left.^{\circledR}\right)$, compared with a pressurised inhaler in a study of terbutaline in asthmatics. Eur Respir J 1988;1:681-4.

21 Johnsen CR, Weeke ER. Turbuhaler ${ }^{\circledR}$ : a new device for dry powder terbutaline inhalation. Allergy 1988;43:392-5.

22 Borgström L, Derom E, Stăhl E, et al. The inhalation devices influences lung deposition and bronchodilating effect of terbutaline. Am J Respir Crit Care Med 1996:153:1636-40.

23 Ekström T, Andersson AC, Skedinger M, et al. Dose potency relationship of terbutaline inhaled via Turbuhaler or via a pressurised metered dose inhaler. Ann Allergy 1995;74:328-32.

24 Nana A, Youngchaiyud P, Maranetra N, et al. $\beta_{2}$-agonists administered by a dry powder inhaler can be used in acute asthma. Respir Med 1998;92:167-72. 